\title{
Computational pharmaceutical analysis of anti-Alzheimer's Chinese medicine Coptidis Rhizoma alkaloids
}

\author{
HONG-JYE HONG ${ }^{1}$, PO-YUAN CHEN ${ }^{2}$, TZU-CHING SHIH ${ }^{3}$, CHE-YEN OU ${ }^{2}$, MIEN-DE JHUO $^{2}$, \\ YEN-YU HUANG ${ }^{2}$, CHIA-HSING CHENG $^{2}$, YU-CHI WU ${ }^{2}$ and JING-GUNG CHUNG ${ }^{2,4}$ \\ ${ }^{1}$ School of Chinese Medicine, College of Chinese Medicine; ${ }^{2}$ Department of Biological Science and Technology, \\ College of Life Sciences; ${ }^{3}$ Department of Biomedical Imaging and Radiological Science, \\ China Medical University; ${ }^{4}$ Department of Biotechnology, Asia University, Taiwan, R.O.C.
}

Received September 15, 2011; Accepted October 12, 2011

DOI: $10.3892 / \mathrm{mmr} .2011 .630$

\begin{abstract}
Alzheimer's disease (AD) is an age-related neurodegenerative disease, affecting over 20 million people worldwide. Until recently, two major hypotheses were proposed regarding the molecular mechanism of pathogenesis: the cholinergic hypothesis and the amyloid cascade hypothesis. At present, acetylcholinesterase inhibitors are the most effective therapy for AD. Most pharmacological research has focused on the ability of acetylcholinesterase to alleviate cholinergic deficit and improve neurotransmission. Coptidis rhizoma and its isolated alkaloids are reported to possess a variety of activities, including neuroprotective and antioxidant effects. However, as yet no theoretical analysis exists to support this hypothesis. To examine this theory, we applied a computational pharmaceutical analysis to reveal that Chinese medicine Coptidis rhizoma alkaloids have much higher activities than Donepezil (commercial name is Aricept) by docking and scoring.
\end{abstract}

\section{Introduction}

Alzheimer's disease (AD), a debilitating disease of the elderly, is the most common cause of senile dementia characterized by a progressive deterioration in cognitive function and loss of memory (1). According to statistics, the number of patients suffering from AD is likely to double in the next 20 years. AD has become a disease that causes heavy social and financial burden to both society and the families involved (2). Until recently, two major hypotheses were proposed regarding the molecular mechanism of pathogenesis: the cholinergic hypothesis and the amyloid cascade hypothesis (3). To treat and prevent $\mathrm{AD}$, most pharmacological research has focused

Correspondence to: Dr Jing-Gung Chung or Dr Po-Yuan Chen, Department of Biological Science and Technology, China Medical University, No. 91, Hsueh-Shih Road, Taichung 404, Taiwan, R.O.C. E-mail: jgchung@mail.cmu.edu.tw; pychen@mail.cmu.edu.tw

Key words: Alzhimer's disease, AChE inhibitors, Coptidis rhizoma alkaloids on acetylcholinesterase (AChE) and butyrylcholinesterase (BChE) inhibitors to alleviate cholinergic deficit and improve neurotransmission (4). Amyloid $\beta$ peptide $(A \beta)$ is the main factor to cause $\mathrm{AD}$ (5-8). Since $\mathrm{A} \beta$ results from the proteolysis of amyloid precursor protein (APP) by $\beta$ - and $\gamma$-secretases, and the formation and accumulation of $\mathrm{A} \beta$ is crucial in AD pathogenesis, the $\beta$-site APP cleaving enzyme 1 (BACE1; aspartyl protease, Asp2 and memapsin2) has recently emerged as a prevalent therapeutic target for AD. The cerebral cholinergic neuron of AD patients has been found to decrease by $30-95 \%$, particularly in the cerebral cortex and Hippocampus.

The drugs used to cure AD, such as Donepezil (commercial name is Aricept), Rivastigmine (commercial name is Exelon) (9) and Galantamine (commercial name is Reminyl) (10) (Fig. 1), are the main products according to the cholinergic hypothesis. These drugs are classifed using the following criteria: i) AD patients cerebral cortex cholinergic neuroticism decreases; ii) AD patients cerebral acetyltransferase activity decreases; iii) insufficient cholinergic is correlated to cognition function; and iv) improvement is noted using $\mathrm{AChE}$ inhibitors.

Two common cholinesterases have usually been discussed in the treatment of AD: AChE, which is mainly found in cerebrum, striated muscle, skin and red blood cells; and BChE (11), which appears in the myocardium, smooth muscle, skin, gland and blood serum. Some drugs are capable of inhibiting combined AChE and BChE, causing serious side effects, such as gastrointestinal discomfort and risk of bradycardia.

According to AChE-Donepezil (PDB code 1EVE), Donepezil interacts with various residues of the AChE active site, which performs interactions with the following groups: benzyl, pieridinic nitrogen and dimethoxy-indanone, which results in $\pi$ stacking and hydrophobic interaction. The binding of Donepezil with AChE is extremely dependent on the interactions with Trp279 and Phe330, which do not appear in BChE. These activities successfully elucidate the reason for the use of Donepezil for a higher specificity of AChE than BChE.

Coptidis rhizoma (the rhizomes of Coptis chinensis FRANCH) is known as 'Huang Lian' and is used in the treatment of various diseases in traditional Chinese medicine due to its anti-diabetic, relaxant, pyretic, antibacterial and antiviral effects (12). Coptidis rhizoma is also known to hold a diversity of alkaloids, including berberine, palmatine, jateorrhizine, 


\section{Donepezil}

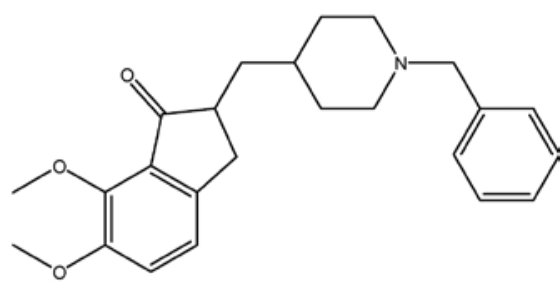

Rrivastigmine<smiles>CCN(C)C(=O)Oc1cccc([C@@H](C)N(C)C)c1</smiles>

\section{Galantamine}

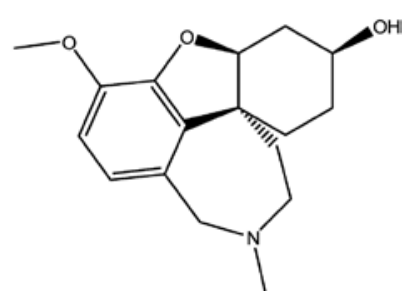

Figure 1. Structures of AChE inhibitors as FDA-approved AD therapeutics.

epiberberine and coptisine (Fig. 2). Additionally, it exerts a variety of activities, including anti-hypertensive, anti-diabetic, anti-inflammatory, hypolipidemic, anti-diabetic complications and antioxidant effects (13). In particular, Coptidis rhizoma and its isolated alkaloids have been reported to exhibit cognitive-enhancing, anti-depressant and cholinesterase-inhibitory effects. Although much research has been conducted regarding anti-AD and the neuroprotective effects of Coptidis rhizoma and its alkaloids (14), the relationship between individual alkaloid structures and anti-AD effects, as well as its relevance to antioxidant effects, remains limited. Furthermore, protoberberine alkaloids with a relatively low molecular weight and high lipophilicity are supposed to meet the requirements of promising therapeutic drugs for AD.

In this study, we assessed the Chinese medicine Coptidis rhizoma alkaloids, which exhibit a much higher Dock score than Donepezil, evaluating the inhibitory effects of isolated alkaloids from Coptidis rhizoma and analyzing the alkaloid structures from the points of physical chemistry.

\section{Materials and methods}

Homology modeling using Accelrys software. Homology modeling was employed to construct an atomic-resolution model of the target protein from its amino acid sequence and an experimental three-dimensional structure of a related homologous protein. It relies on the identification of one or more known protein structures likely to resemble the structure of the query sequence. The target structure is produced from the known sequence alignment and the template structure and its sequence. Since protein structures are more conserved than DNA sequences, detectable levels of sequence similarity usually indicate significant structural similarity.

Dock score using Accelrys software. The score functions in the Discovery Studio 2.5 that we used were Dock score,<smiles>COc1ccc2cc3[n+](cc2c1OC)CCc1cc2c(cc1-3)OCO2</smiles><smiles>COc1cc2c(cc1O)CC[n+]1cc3c4c(ccc3cc1-2)OCO4</smiles><smiles>COc1cc2c(cc1O)CC[n+]1cc3c(OC)c(OC)ccc3cc1-2</smiles><smiles></smiles><smiles>COc1cc2c(cc1OC)-c1cc3ccc(OC)c(OC)c3c[n+]1CC2</smiles><smiles>COc1cc2c(cc1OC)-c1cc3ccc4c(c3c[n+]1CC2)OCO4</smiles>

Figure 2. Structure of alkaloids from Coptidis rhizoma (continued). 
piecewise linear potential (PLP) 1, PLP2 and PMF. Candidate ligand poses are evaluated and prioritized according to the Dock score function. There are two types of Dock score. One is based on a forcefield approximation, and the other on the PLP function:

Equation 1: Dock score $($ forcefield $)=-($ ligand $/$ receptor interaction energy + ligand internal energy).

Equation 2: Dock score (PLP) $=$ - (PLP potential).

As shown in Equation 1, there are two energy terms in the forcefield version of the Dock score: internal energy of the ligand and interaction energy of the ligand with the receptor. The interaction energy is taken as the sum of the van der Waals energy and electrostatic energy. However, the computation of the interaction energy can be time-consuming. Thus, to reduce the time required for this calculation, a grid-based estimation of the ligand/receptor interaction energy is employed.

The van der Waals component of the forcefield interaction energy typically exhibits a steep rise at short interatomic distances, which may have undesirable consequences in the context of ligand-receptor docking. In particular, the combination of approximating the receptor structure as a rigid and limited sampling of ligand conformational space tends to overly penalize poses with 'mild' short contacts between the ligand and receptor, due to the 'hard' nature of the van der Waals potential as defined in the majority of standard forcefields.

To overcome this tendency, a softened form of the van der Waals potential is employed with the Dock score function. This softened potential rises to a large but finite value at zero interatomic separation. To maintain a proper balance between electrostatics and van der Waals, electrostatic energy is also softened to prevent it from dominating the van der Waals energy at short separations.

The internal energy of the ligand is computed when using the forcefield version of Dock score. The purpose of including the internal energy is to avoid ligand conformations with bad internal non-bond clashes. By default, only the standard (not softened) van der Waals energy is used for the ligand internal energy. The inclusion of electrostatic energy as part of the ligand internal energy is optionally available.

The PLP version of Dock score uses the PLP1 function, because the functional form of PLP1 allows it to be readily represented with a grid-based approach. The PLP2 function has an angular dependence on hydrogen bonding interactions making its representation with a grid considerably more difficult.

In the PLP1 score function, there are four atom types: i) hydrogen bond donor only, ii) hydrogen bond acceptor only, iii) both hydrogen bond donor and acceptor, and iv) non-polar. When PLP1 is the Dock score function, the internal energy is calculated for each ligand conformation for which the ligand is in the binding site.

In the PLP2 score function, the atom typing remains the same as in the PLP1 score function. In addition, an atomic radius is assigned to each atom with the exception of hydrogen (15).

The PMF score function was developed based on a statistical analysis of the three-dimensional structures of the protein-ligand complex (16). These structures were found to correlate well with protein-ligand binding-free energy, while being fast and simple to calculate. The scores are calculated by summing pairwise interaction terms over all interatomic pairs of the receptor-ligand complex. The score function of Dock score is the default function in the Discovery Studio 2.5.

All the simulations were also applied by the Forcefield of Chemistry at Chemistry at Harvard Macromolecular Mechanics (CHARMM). CHARMM was parameterized by experimental data. It has been used widely for simulations ranging from small molecules to solvated complexes of large biological macromolecules. CHARMM performs well over a broad range of calculations and simulations, including minima, time-dependent dynamic behavior and barriers to rotation, vibrational frequencies and free energy. CHARMM uses a flexible and comprehensive energy function: $\mathrm{E}_{(\mathrm{pot})}=$ $\mathrm{E}_{\text {bond }}+\mathrm{E}_{\text {torsion }}+\mathrm{E}_{\text {oop }}+\mathrm{E}_{\text {elect. }}+\mathrm{E}_{\mathrm{vdW}}+\mathrm{E}_{\text {constraint }}+\mathrm{E}_{\text {user }}$, where the out-of-plane (OOP) angle is an important torsion. The van der Waals term is derived from rare-gas potentials, and the electrostatic term can be scaled to mimic solvent effects. Hydrogen-bond energy is not included as a separate term as in AMBER. Instead, hydrogen-bond energy is implicit in the combination of van der Waals and electrostatic terms.

De novo evolution using Accelrys software. To design the new compounds from the potent drugs, we used the program 'De novo evolution' in the Discovery Studio 2.5 (Accelrys, San Diego, CA, USA). The new compounds were transfected from the de novo evolution into the protein acceptor binding pocket and the scores were calculated.

ADMET descriptors using Acclery Software. We computed the adsorption, distribution, metabolism, excretion and toxicity (ADMET) properties of small molecules using Discovery Studio 2.5 (Accelrys) to estimate the following properties: aqueous solubility blood brain barrier (BBB) penetration, cytochrome P450 (CYP450) 2D6 inhibition, hepatotoxicity human intestinal absorption (HIA) and plasma protein binding. Furthermore, calculating the BBB and other factors was crucial as the drugs are required to pass through the $\mathrm{BBB}$ to react with the receptor protein in order to heal AD.

This model predicts BBB penetration after oral administration. Moreover, this model contains a quantitative linear regression model for the prediction of blood-brain penetration, as well as 95 and $99 \%$ confidence ellipses in the ADMET_ PSA_2D, ADMET_AlogP98 plane. These ellipses are not the same as those associated with the ADMET - human intestinal absorption, although they have an analogous interpretation. They were derived from over 800 compounds that are known to enter the CNS after oral administration.

\section{Results and Discussion}

The enzyme monomer of the T. californica AChE (PDB accession code: $1 \mathrm{EVE}$ ) is an $\alpha / \beta$ serine hydrolase consisting of 537 residues with a 12-strand mixed $b$ sheet surrounded by $14 \alpha$ helices (17). The active site is similar to approximately $20 \AA$ above the narrow tunnel. This tunnel penetrates halfway into the enzyme and widens out at the end. Fourteen highly conserved residues cover a substantial portion of the tunnel. The button of the tunnel, approximately $4 \mathrm{~A}$ long, is the catalytic machinery, i.e., Ser200, His440 and Glu327 formed a catalyst. In the region of the enzymatic cavity located 


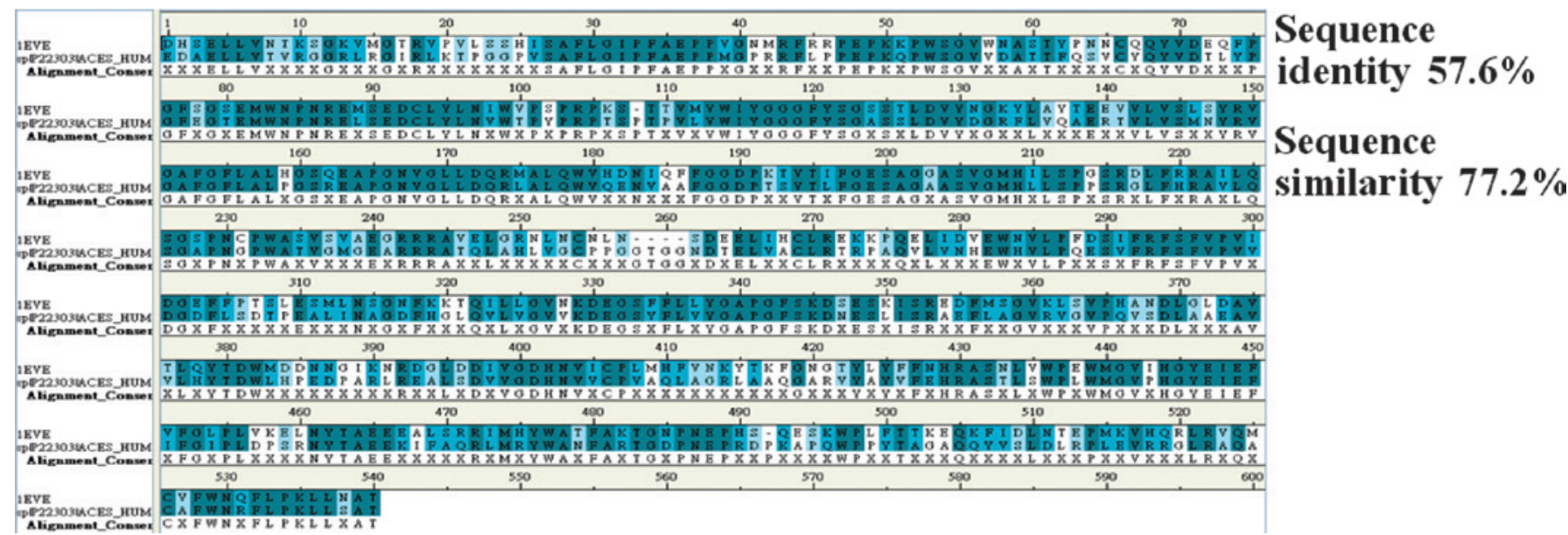

Figure 3. Results of multiple sequence alignment. Sequence similarity was $77.2 \%$ and sequence identity was $57.6 \%$.

A

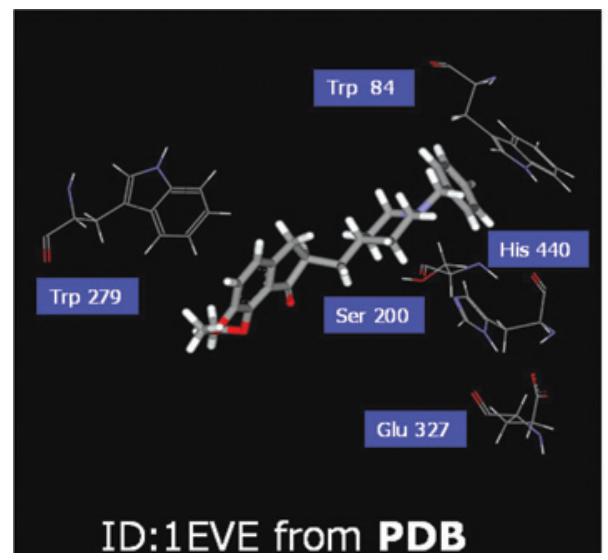

B

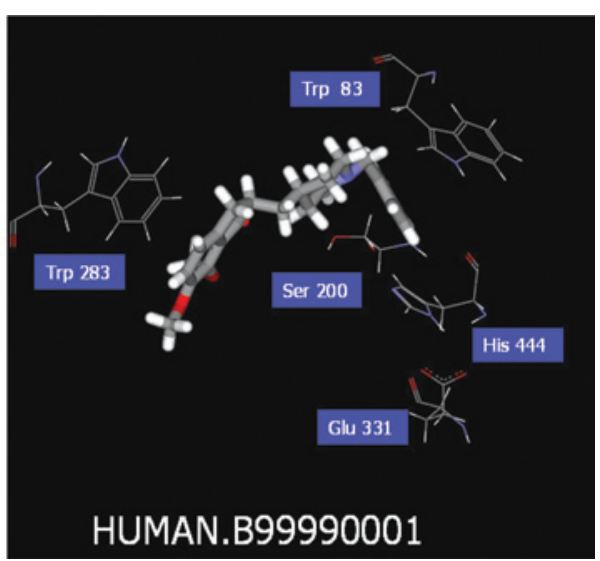

Figure 4. (A) Donepezil in crystal structure 1EVE. (B) Crystal structure binding mode of Donepezil in the active site of the homology model. Key residues, Ser 200, His 440, Glu 327, Trp 84 and Trp 279 convert into Ser 200, His 444, Glu 331, Trp 83 and Trp 283.

Table I. Score values of new AChE inhibitors and Donepenzil.

\begin{tabular}{lccc}
\hline & -PLP1 & -PMF & Dock Score \\
\hline Donepenzil & 78.52 & 153.85 & 67.85 \\
Berberin & 91.83 & 178.46 & 97.46 \\
Coptisine & 72.63 & 217.56 & 95.16 \\
Epiberberine & 73.25 & 217.68 & 89.47 \\
Groenlandicine & 63.95 & 141.65 & 89.70 \\
Palmatine & 73.55 & 194.93 & 97.11 \\
\hline
\end{tabular}

between the choline binding and peripheral anionic sites, the phenyl group of Phe330 of the enzymatic cavity interacts by cation- $\pi$ with the piperidinic nitrogen of the Donepezil, which contains a positive charge, while Tyr334 forms a calix-domain with Phe330 and the piperidinic ring of Donepezil. Thus, the interaction with Phe330 is an additional site of quantaernary binding with functional significance, inside the active site. In the peripheral anionic site, the indanone ring interacts with the indonic group of Trp279, via a classical parallel $\pi-\pi$ interaction. Recent data suggest that the inhibitors interacting with the peripheral anionic site may prevent the A from interacting

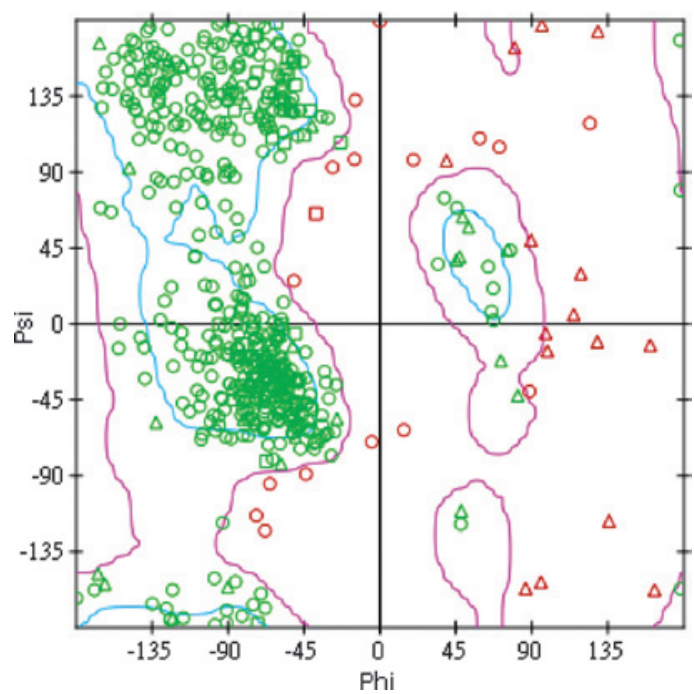

Figure 5. Ramchandran plot analysis showed $78.2 \%$ of the residues in the most favored region, $16.1 \%$ in the allowed and $5.7 \%$ in the disallowed region.

with AChE. This may result in the elimination of AChE involvement in the fibril formation process (18).

In order to make the structure of AChE closer to that of humans, the three-dimensional homology model of the AChE 
A

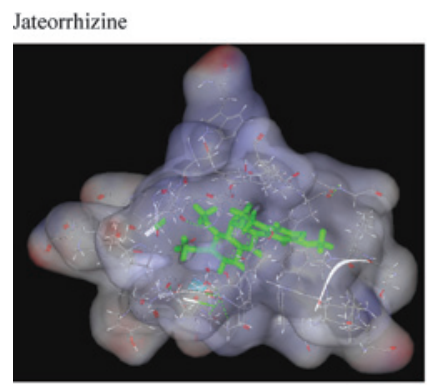

Coptisine

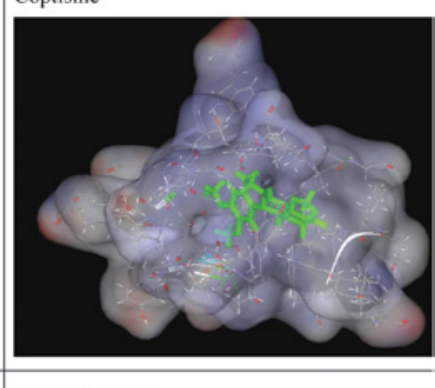

Epiberberine

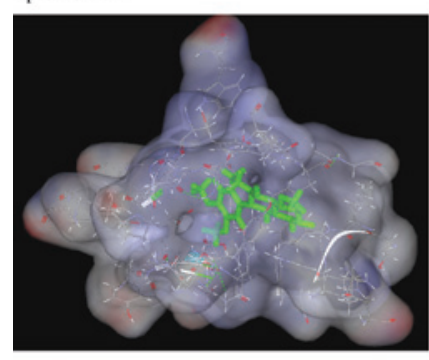

Groenlandicine

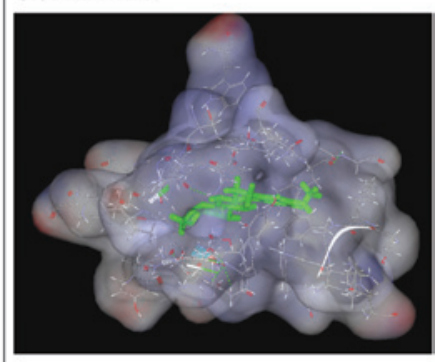

Berberine

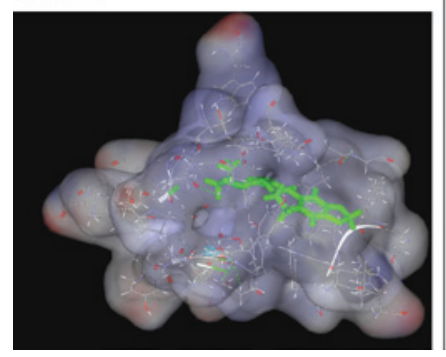

Palmatine

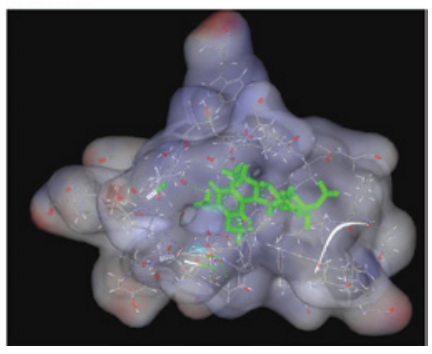

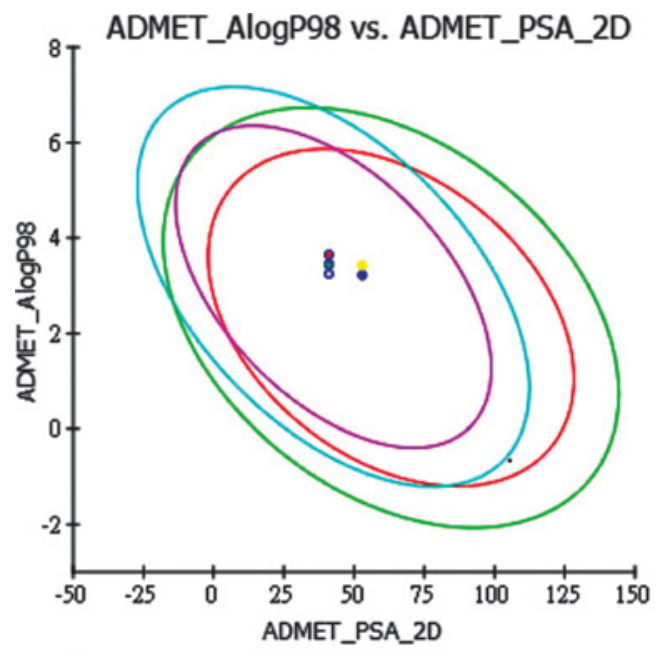
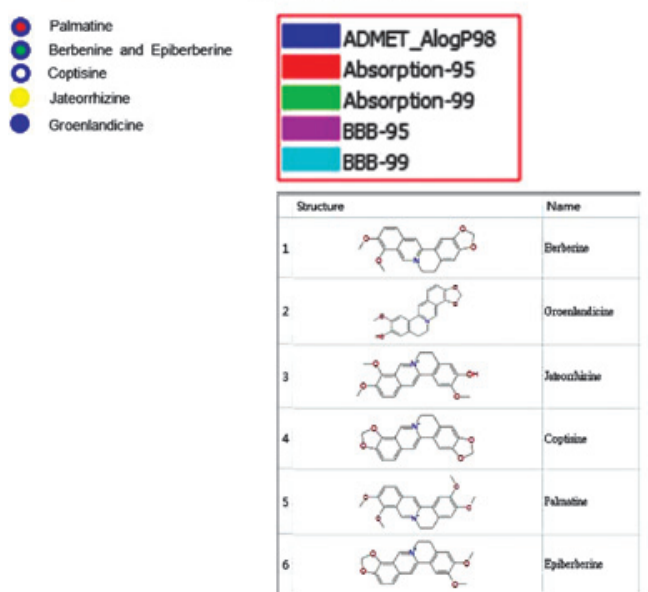

Figure 6. The docking-active site of Coptidis rhizoma alkaloids is shown.

of human was established using the Discovery Studio 2.5 (Accelrys) from the crystal structure of the $T$. californica AChE (PDB accession code: 1EVE) and the known sequence (P04058 from Swiss-Prot). To perform multiple sequence alignment, framework construction, energy minimization and structure reasonable analysis, we constructed human AChE by homology modeling. The human AChE exhibited a high sequence similarity (Fig. 3) with the crystal structure of the T. californica AChE. This successfully indicated that we were able to simulate the structures precisely.

We constructed the structure of HUMAN.B99990001, which is a result of the homology model with the Discovery Studio 2.5 and which contained key residues. We found that Ser 200, His 440, Glu 327, Trp 84 and Trp 279 convert into Ser 200, His 444, Glu 331, Trp 83 and Trp 283 (Fig. 4). Energy minimization was then performed using molecular mechanics energy minimization to correct unfavorable non-covalent touch and to reach ideal bond geometry and the lowest energy configuration. The Ramchandran plot analysis showed 78.2\% of the residues in the most favored region, $16.1 \%$ in the allowed and $5.7 \%$ in the disallowed region (Fig. 5). Donepezil docking to HUMAN.B99990001 revealed that Donepezil combines to the tunnel of AChE. Trp 279 and Phe 330 still formed the $\pi-\pi$ interaction with HUMAN.B99990001 (Fig. 6A).

We also performed ADMET using Discovery Studio 2.0 (Accelrys). A two-dimentional ADMET plot showed that the six compounds pass through the $\mathrm{BBB}$, and affect the brain (Fig. 6B).

In conclusion, the traditional Chinese medicine Coptidis rhizoma alkaloids has been found to have a much higher Dock score than Donepezil, due to its hydrophobic ring system, and has a low molecular weight. The compounds pass through the BBB and affect the brain. On the other hand, the Coptidis rhizoma is cost-effective, and the extracts of the herb may prove a cheaper way of treating AD. The costs of drug production and synthesis may also be significantly decreased. These docking studies provide data regarding the manner in which the inhibitors interact with target proteins. However, further studies are required to design more potent drugs for the treatment of $\mathrm{AD}$.

\section{Acknowledgements}

The authors acknowledge the support of the China Medical University in this study.

\section{References}

1. Dorronsoro C, Barbero S, Llorente L, et al: On-eye measurement of optical performance of rigid gas permeable contact lenses based on ocular and corneal aberrometry. Optom Vis Sci 80: $115-125,2003$.

2. Guo T and Hobbs DW: Development of BACE1 inhibitors for Alzheimer's disease. Curr Med Chem 13: 1811-1829, 2006. 
3. Parihar MS and Hemnani T: Alzheimer's disease pathogenesis and therapeutic interventions. J Clin Neurosci 11: 456-467, 2004.

4. Rao AA, Sridhar GR and Das UN: Elevated butyrylcholinesterase and acetylcholinesterase may predict the development of type 2 diabetes mellitus and Alzheimer's disease. Med Hypotheses 69: 1272-1276, 2007.

5. Wood KE, Becker BN, McCartney JG, et al: Care of the potential organ donor. N Engl J Med 351: 2730-2739, 2004.

6. Jacobsen JS, Reinhart P and Pangalos MN: Current concepts in therapeutic strategies targeting cognitive decline and disease modification in Alzheimer's disease. NeuroRx 2: 612-626, 2005.

7. Mori F, Fukaya M, Abe H, et al: Developmental changes in expression of the three ryanodine receptor mRNAs in the mouse brain. Neurosci Lett 285: 57-60, 2000.

8. Barril X, Orozco M and Luque FJ: Towards improved acetylcholinesterase inhibitors: a structural and computational approach. Mini Rev Med Chem 1: 255-266, 2001.

9. Jann MW: Rivastigmine, a new-generation cholinesterase inhibitor for the treatment of Alzheimer's disease. Pharmacotherapy 20: 1-12, 2000.

10. Zarotsky V, Sramek JJ and Cutler NR: Galantamine hydrobromide: an agent for Alzheimer's disease. Am J Health Syst Pharm 60: 446-452, 2003.

11. Hshieh TT, Fong TG, Marcantonio ER, et al: Cholinergic deficiency hypothesis in delirium: a synthesis of current evidence. J Gerontol A Biol Sci Med Sci 63: 764-772, 2008.
12. Huang KC (ed.): The Pharmacology of Chinese Herbs. CRC Press Inc., Boca Raton, 1999.

13. Kim DK, Lee KT, Baek NI, et al: Acetylcholinesterase inhibitors from the aerial parts of Corydalis speciosa. Arch Pharm Res 27: $1127-1131,2004$

14. Asai M, Iwata N, Yoshikawa A, et al: Berberine alters the processing of Alzheimer's amyloid precursor protein to decrease Abeta secretion. Biochem Biophys Res Commun 352: 498-502, 2007.

15. Bouzida D, Arthurs S, Colson AB, et al: Thermodynamics and kinetics of ligand-protein binding studied with the weighted histogram analysis method and simulated annealing. Pac Symp Biocomput: 426-437, 1999.

16. Muegge I and Martin YC: A general and fast scoring function for protein-ligand interactions: a simplified potential approach. J Med Chem 42: 791-804, 1999.

17. Sussman JL, Harel M, Frolow F, et al: Atomic structure of acetylcholinesterase from Torpedo californica: a prototypic acetylcholine-binding protein. Science 253: 872-879, 1991.

18. Ferrari D, Yang LH, Miles EW, et al: Beta D305A mutant of tryptophan synthase shows strongly perturbed allosteric regulation and substrate specificity. Biochemistry 40: 7421-7432, 2001. 